\title{
Antihyperlipidaemic and hepatoprotective activities of acidic and enzymatic hydrolysis exopolysaccharides from Pleurotus eryngii SI-04
}

Chen Zhang ${ }^{1,2 \dagger}$, Juan $\mathrm{Li}^{3 \dagger}$, Jing Wang ${ }^{4}$, Xingling Song ${ }^{2}$, Jianjun Zhang ${ }^{2}$, Shang $\mathrm{Wu}^{5}$, Chunlong $\mathrm{Hu}^{6}$, Zhiyuan Gong ${ }^{1 *}$ and Le Jia ${ }^{2^{*}}$

\begin{abstract}
Background: Hyperlipidaemia is the major risk factor contributing to the development and progression of atherosclerosis, fatty liver and cerebrovascular disease. Pleurotus eryngii (P. eryngii) is rich in biologically active components, especially polysaccharides that exhibit various biological activities, including reducing blood lipids. In the present study, three novel polysaccharide types, including exopolysaccharides (EPS), enzymatic EPS (EEPS) and acidic EPS (AEPS) were isolated, and the hypolipidaemic and hepatoprotective effects were investigated to better understand possible hypolipidaemic mechanisms and their hepatoprotective effects.

Methods: The EPS was hydrolysed by snailase (dissolved in $1 \%$ acetic acid, $\mathrm{pH}=6$ ) and $\mathrm{H}_{2} \mathrm{SO}_{4}(1 \mathrm{M}$ ) to obtain EEPS and AEPS, respectively. The in vitro antioxidant activities were measured by investigating the reducing power and the scavenging effects on radicals of hydroxyl, 1,1-diphenyl-2-picrylhydrazyl (DPPH) and superoxide anion. The hyperlipidaemic mice were induced by perfusing a high-fat emulsion. In addition to the hepatic histopathology, the following biochemical analyses were performed to investigate the antioxidative effects, including the activities of alkaline phosphatase (ALP), alanine aminotransferase (ALT), aspartate aminotransferase (AST), glutathione peroxidase (GSH-Px), superoxide dismutase (SOD) and catalase (CAT). Triacylglycerol (TG), total cholesterol (TC), high-density lipoprotein cholesterol (HDL-C), low-density lipoprotein cholesterol (LDL-C), malondialdehyde (MDA) and lipid peroxidation (LPO) levels were also measured in serum and liver homogenate.

Results: Supplementation of EPS, EEPS and AEPS could significantly improve blood lipid levels (TC, TG, HDL-C, and LDL-C), hepatic lipid levels (TC and TG), hepatic enzyme activities (ALP, ALT, and AST) and antioxidant status (GSHPx, SOD, T-AOC, MDA, and LPO). In addition, histopathological observations indicated that these polysaccharides had potential effects in attenuating hepatocyte damage.

(Continued on next page)
\end{abstract}

\footnotetext{
*Correspondence: sdgzy2656@126.com; sdgzy2656@126.com.cn; jiale0525@163.com

${ }^{\dagger}$ Equal contributors

${ }^{1}$ Institute of Agricultural Resources and Environment, Shandong Academy of

Agricultural Science, Key Laboratory of Wastes Matrix Utilization, Ministry of

Agriculture, Jinan 250100, China

${ }^{2}$ College of Life Science, Shandong Agricultural University, Taian 271018,

China

Full list of author information is available at the end of the article
} International License (http://creativecommons.org/licenses/by/4.0/), which permits unrestricted use, distribution, and reproduction in any medium, provided you give appropriate credit to the original author(s) and the source, provide a link to the Creative Commons license, and indicate if changes were made. The Creative Commons Public Domain Dedication waiver (http://creativecommons.org/publicdomain/zero/1.0/) applies to the data made available in this article, unless otherwise stated. 
(Continued from previous page)

Conclusion: These results demonstrated that both EPS and its hydrolysates EEPS and AEPS might effectively reduce serum lipid levels and protect against high-fat diet-induced hyperlipidaemia, indicating that they could be used as functional foods and natural hepatoprotectants.

Keywords: Antihyperlipidaemic, Hepatoprotective, Antioxidant, Acidic and enzymatic hydrolysis exopolysaccharides, Pleurotus eryngii SI-04

\section{Background}

High-fat diets provide excess energy intake and disrupt lipid metabolism, resulting in fat accumulation in many tissues, especially serum and liver [1]. Hyperlipidaemia, always caused by high-fat diets, is mainly characterized by increased levels of total cholesterol (TC), triglyceride (TG) and low-density lipoprotein cholesterol (LDL-C), along with a decrease in high-density lipoprotein cholesterol (HDL-C). These changes are the major risk factors contributing to the development and progression of atherosclerosis, fatty liver and cerebrovascular disease [2-5]. Many researchers have focused on the roles of oxidative damage and lipid peroxidation in the pathomechanism of hyperlipidaemia [6]. Oxidative stress, always caused by superfluous reactive oxygen species (ROS), is an early event in the evolution of hyperlipidaemia. ROS, including hydroxyl (HO.), DPPH. and superoxide $\left(\mathrm{O}_{2}^{-}\right.$.) radicals, are potentially toxic to various biological molecules, resulting in oxidative damage that can accelerate the pathogenic progress of hyperlipidaemia and its complications [6-8]. Under hyperlipidaemic conditions, enzymatic and non-enzymatic antioxidative defence systems such as superoxide dismutase (SOD), catalase (CAT) and glutathione peroxidase (GSH-Px) are altered, leading to ROS-mediated damage [9]. Scientists have suggested that the appropriate support for enhancing the antioxidant supply in subjects with hyperlipidaemia can attenuate the course of the disease. Maladjusted lipid synthesis and lipid clearance also play roles in causing hyperlipidaemia, and methods to reduce blood lipid levels could be effective in treating this disease [5]. Thus, effects on the antioxidant and hypolipidaemic properties of some bioactive compounds are particularly promising for improving human health [10].

Recently, the treatment of hyperlipidaemia has been involved in diet control, exercise and pharmaceutical therapy. Since synthetic lipid-lowering drugs, including statins and fibrates, usually have side effects and contraindications with long-term use, the application of natural hypolipidaemic drugs seems to be urgent to prevent and treat hyperlipidaemia and its complications [11]. Pleurotus eryngii (P. eryngii), one type of common edible fungus in China, is rich in biologically active components, including polysaccharides, peptide, sterols and dietary fibre [12]. As the most potent mushroom-derived substances, polysaccharides exhibit various biological activities, including antioxidant, anti-aging, antivirus and anti-lipid peroxidation properties. [13]. Furthermore, modified polysaccharides have received more attention due to their superior physicochemical properties, including good water-solubility, high stability, and non-toxicity [14]. Previous studies have shown that crude polysaccharides from the fruiting body of $P$. eryngii have potential effects in reducing blood lipids [15]. However, the hypolipidaemic effects of exopolysaccharides and their chemically modified forms have not been evaluated. In the present study, three kinds of novel polysaccharides - exopolysaccharides (EPS), enzymatic EPS (EEPS) and acidic EPS (AEPS) - were isolated, and their hypolipidaemic and hepatoprotective effects were investigated. EPS, EEPS and AEPS possessed hypolipidaemic and antioxidant activities, indicating that the polysaccharides could be developed as valuable functional foods/drugs for clinical hypolipidaemic and hepatoprotective treatments.

\section{Methods}

\section{Strain and chemicals}

The P. eryngii SI-04 strain was provided by the Fungi Institute of the Academy of Agricultural Sciences (Tai'an, China). The diagnostic kits for analysing SOD activities, GSH-Px activities, CAT activities, total antioxidant capacity (T-AOC) activities, lipid peroxidation (LPO) contents and malondialdehyde (MDA) contents were purchased from the Nanjing Jiancheng Bioengineering Institute (Nanjing, China). The standard monosaccharide samples, including rhamnose (Rha), ribose (Rib), arabinose (Ara), xylose (Xyl), glucose (Glc), mannose (Man) and galactose (Gal) were provided by the Merck Company (Darmstadt, Germany) and Sigma Chemical Company (St. Louis, USA). Other reagents and chemicals used in the present work were analytical reagent grade and were supplied by local chemical suppliers.

\section{Preparation of EPS}

The liquid fermentation of $P$. eryngii SI-04 was processed using the method from our present work [16]. The EPS of $P$. eryngii SI-04 was obtained by referencing the method of Ma et al. (2015) with slight modifications. After centrifugation (3000 rpm, $15 \mathrm{~min}$ ), the supernatant fermentation broth was mixed with 3 volumes of $95 \%$ 
ethanol $(v / v)$, stirred thoroughly and stored at $4{ }^{\circ} \mathrm{C}$ for $24 \mathrm{~h}$. The precipitate was deproteinized with Sevag reagent (chloroform/n-butanol, 5:1, v/v) and lyophilized by vacuum freeze-drying (Labconco, USA) to obtain EPS. The EPS was weighed, and the yield was $3.81 \mathrm{~g} / \mathrm{L}$.

\section{Enzymatic and acidic hydrolysis of EPS}

The enzymatic hydrolysis of EPS was processed according to the methods of Yang et al. [17] and Li et al. [18] with some modifications. The polysaccharide sample (0.5 g) and snailase $(0.1 \mathrm{~g})$ were dissolved in $100 \mathrm{~mL}$ of $1 \%$ acetic acid at $\mathrm{pH} 6$ and $37^{\circ} \mathrm{C}$ for $4 \mathrm{~h}$. After quick pre-freezing, the enzymatic hydrolysis exopolysaccharides (EEPS) were lyophilized for further analyses.

The acidic hydrolysis of EPS was processed according to the method of $\mathrm{Ma}$ et al. [19] with slight modifications. Briefly, EPS (0.5 g) was dissolved in $10 \mathrm{~mL}$ of $1 \mathrm{M} \mathrm{H}_{2} \mathrm{SO}_{4}$ solution, and the reaction was processed in a boiling water bath for $8 \mathrm{~h}$. After centrifugation (6000 rpm, $10 \mathrm{~min}$ ) and neutralization, the supernatant was concentrated and lyophilized to obtain acidic exopolysaccharides (AEPS).

\section{Monosaccharide composition analysis}

The monosaccharide compositions of EPS, EEPS and AEPS were calculated using gas chromatography (GC2010, Shimadzu, Japan) equipped with a flame ionization detector (FID) and an Rtx-1 capillary column $(30 \mathrm{~m} \times 0.25 \mathrm{~mm} \times 0.25 \mu \mathrm{m})$. The samples and standard monosaccharides were pre-processed using our previous method [16]. The initial oven temperature of the column was maintained at $190{ }^{\circ} \mathrm{C}$ for $20 \mathrm{~min}$ and increased gradually to $200{ }^{\circ} \mathrm{C}$ at a rate of $3{ }^{\circ} \mathrm{C} / \mathrm{min}$. Nitrogen was used as the carrier gas at $0.8 \mathrm{~mL} / \mathrm{min}$ of cavity flow and $19.8 \mathrm{~mL} / \mathrm{min}$ of total flow. The samples $(1.0 \mu \mathrm{L})$ were injected in the split model $(1: 20)$ at $260{ }^{\circ} \mathrm{C}$. The monosaccharide content was expressed as the following formula:

$$
\text { Monosaccharide content }(\%)=\frac{A}{B} \times \frac{V}{M} \times C
$$

where A and B were the peak areas of sample and standard monosaccharides, $\mathrm{V}$ was the sample constant volume $(\mathrm{mL}), \mathrm{M}$ was the sample quality $(\mathrm{g})$, and $\mathrm{C}$ was the monosaccharide concentration of the mixed standard $(\mathrm{mg} / \mathrm{mL})$.

\section{Antioxidant effects in vitro}

The reducing power was assayed according to our previous work [16].

The scavenging capability on hydroxyl radicals was evaluated using the method of Koksal et al. [20] with few modifications. The reaction mixture, including $1 \mathrm{~mL}$ of phenanthroline $(7.5 \mathrm{mM}), 1 \mathrm{~mL}$ of ferrous sulphate
(0.75 mM), $5 \mathrm{~mL}$ of phosphate buffer $(\mathrm{pH} 7.4), 1 \mathrm{~mL}$ of sample $(0-1000 \mathrm{mg} / \mathrm{L})$ and $1 \mathrm{~mL}$ of hydrogen peroxide $(3 \%, v / v)$ was shaken sufficiently and incubated at $37{ }^{\circ} \mathrm{C}$ for $30 \mathrm{~min}$. The absorbance was measured at $560 \mathrm{~nm}$ using distilled water as a blank, and the scavenging rate was calculated using the following formula:

$$
\text { Scavenging rate }(\%)=\frac{A-B}{B} \times 100
$$

where A was the absorbance of distilled water, and B was the absorbance of samples.

The scavenging capability on DPPH radicals was measured using the methods of Brand-Williams et al. [21] and Kong et al. [22] with some modifications. The reaction mixture, containing $2 \mathrm{~mL}$ of ethanol $(95 \%, w / v), 0.1 \mathrm{~mL}$ of $\mathrm{DPPH}(\mathrm{l} \mathrm{M})$ and $2 \mathrm{~mL}$ of sample $(0-1000 \mathrm{mg} / \mathrm{L})$, was incubated at room temperature and placed in the dark for $30 \mathrm{~min}$. The absorbance of the solution was determined at $517 \mathrm{~nm}$. The scavenging rate was evaluated using the following formula:

$$
\text { Scavenging rate }(\%)=\left(1-\frac{A}{B}\right) \times 100
$$

where A was the absorbance of the tested sample, and B was the absorbance of the blank.

The scavenging capability of superoxide anion radicals was measured using the method of Stewar and Beewley [23] with slight modification. Briefly, $1.0 \mathrm{~mL}$ of sample (0-1000 $\mathrm{mg} / \mathrm{L})$ was added to the mixture containing phosphate-buffered saline $(0.5 \mathrm{~mL}, 0.2 \mathrm{M}, \mathrm{pH} 7.8)$, riboflavin $(0.3 \mathrm{~mL}, 10 \mathrm{mM})$ and methionine $(0.25 \mathrm{~mL}, 13 \mathrm{mM})$, and the reaction was incubated at $25{ }^{\circ} \mathrm{C}$ for $30 \mathrm{~min}$. The absorbance of the solution was determined at $560 \mathrm{~nm}$, and the scavenging rate was calculated using the following formula:

$$
\text { Scavenging rate }(\%)=\left(1-\frac{A}{B}\right) \times 100
$$

where A was the absorbance of polysaccharide samples, and $\mathrm{B}$ was the absorbance of the blank.

\section{Experimental design \\ Preparation of high-fat emulsion}

The high-fat emulsion was prepared using the method of Zhao, Huang and Yuan [11] with sight modifications. Briefly, the oil phase, including $25 \mathrm{~g}$ lard oil, $10 \mathrm{~g}$ cholesterol, $1 \mathrm{~g}$ methylthiouracil and $25 \mathrm{~mL}$ of Tween-80, was heated to the melting point on a magnetic stirring apparatus (Guohua Instrument Ltd. Co. Changzhou, China). Simultaneously, the water phase contained $30 \mathrm{~mL}$ distilled water, $20 \mathrm{~mL}$ propylene glycol and $2 \mathrm{~g}$ sodium deoxycholate. Subsequently, the water and oil phases were mixed thoroughly before animal administration. 


\section{Design of the animal experiment}

Seventy-two Kunming strain mice (20 \pm 2 g, male), purchased from Taibang Biological Products Ltd. Co. (Tai'an, China) were housed in polycarbonate cages and freely accessed food and water ad libitum at constant conditions of $22 \pm 1{ }^{\circ} \mathrm{C}$ and constant humidity (50 $\left.\pm 5 \%\right)$ under a 12 $\mathrm{h}$ light-dark cycle.

After adapting to the environment for $7 \mathrm{~d}$, all mice were weighed and randomly distributed into nine groups (eight mice per group). In the hyperlipidaemia group (HL), mice were perfused with high-fat emulsion alternated with distilled water. Mice in the simvastatin group (ST) were perfused with high-fat emulsion alternated with simvastatin $(200 \mathrm{mg} / \mathrm{kg}$ body weight). In the other six treatment groups (L-EPS, HEPS, L-EEPS, H-EEPS, L-AEPS and H-AEPS), mice were perfused with high-fat emulsion alternated with EPS, EEPS and AEPS at 400 and $800 \mathrm{mg} / \mathrm{kg}$ body weight. Mice in the normal control group (NC) were given distilled water daily, and the entire experiment lasted 28 days. All the experiments were submitted to and approved by the ethics committee of the Shandong Agricultural University.

After overnight fasting, all mice were weighed and sacrificed under anaesthesia. The serum was obtained by centrifugation $(10,000 \mathrm{rpm}, 10 \mathrm{~min})$ from blood in the retrobulbar vein. The livers were excised, weighed and homogenized (1:9, $w / v$, in normal saline and ethyl alcohol). After centrifugation (5000 rpm, $20 \mathrm{~min}, 4{ }^{\circ} \mathrm{C}$ ), the supernatants were collected and stored at $0{ }^{\circ} \mathrm{C}$ for further biochemical analysis.

\section{Biochemical and histopathological assays}

Alkaline phosphatase (ALP), alanine aminotransferase (ALT) and aspartate aminotransferase (AST) activities and TG, TC, HDL-C and LDL-C levels in serum were measured using an automatic biochemical analyser (ACE, USA). GSH-Px, SOD and CAT activities in serum/liver homogenate and the MDA, LPO, TC and TG contents in liver homogenate were analysed using commercial kits according to the instructions.

The liver tissue staining method followed a previously published study [16].

\section{Acute toxicity assay}

The acute toxicity test in mice was performed on the basis of the reported method [24] with some modifications. The mice were randomly divided into four groups, including one control group and three dose groups (eight mice per group). The mice in dose groups received intragastric administration with EPS, EEPS and AEPS at $5000 \mathrm{mg} / \mathrm{kg}$ body weight, while the control group received isometric saline solutions. All mice had free access to food and water ad libitum for 10 days under regular observation for any mortality or behavioural changes, including irritation, restlessness, respiratory distress, abnormal locomotion and catalepsy.

\section{Statistical analysis}

All data are presented as the means \pm standard deviations (SD) of three independent experiments. Significant differences among groups were determined by one-way ANOVA (SPSS 16.0 software package, USA). $P<0.05$ was considered statistically significant.

\section{Results}

\section{Monosaccharide composition analysis}

The monosaccharide compositions of EPS, EEPS and AEPS were identified according to the retention times and chromatograph peaks using monosaccharide guide samples (Fig. 1a). The EPS consisted of five different monosaccharides, including Ara, Xyl, Man, Gal and Glc, in mass percentages of $3.42 \%$, $4.76 \%, 17.03 \%, 4.46 \%$ and $70.32 \%$, with a molar ratio of 1:1.5:4.3:1.1:17.7 (Fig. 1b). The EEPS consisted of three different monosaccharides, including Man, Gal and Glc, in mass percentages of $12.85 \%, 4.93 \%$ and 82.22\%, with a molar ratio of 1:4:1.9:31.2 (Fig. 1c). The AEPS consisted of five monosaccharides, including Ara, Xyl, Man, Gal and Glc, at mass percentages of $1.36 \%, 2.86 \%, 14.50 \%, 2.99 \%$ and $78.03 \%$, with a molar ratio of 1:2.1:15.7:1.8:47.7 (Fig. 1d).

\section{Antioxidant effects in vitro}

In this study, the in vitro antioxidant capacities of EPS, EEPS and AEPS were estimated with reducing power using the HO, DPPH. and $\mathrm{O}_{2}^{-}$. systems. As shown in Fig. $2 \mathrm{a}$, the polysaccharides could cause the reduction of the $\mathrm{Fe}^{3+} / \mathrm{K}_{3} \mathrm{Fe}(\mathrm{CN})_{6}$ complex to $\mathrm{Fe}^{2+}$, as monitored by measurement of the enhanced formation of Perl's Prussian blue at $700 \mathrm{~nm}$ [25]. In the broad range of $100-1000 \mathrm{mg} / \mathrm{L}$, the reducing power of AEPS ranged from $0.152 \pm 0.016$ to $0.817 \pm 0.025$, which was much higher than those of EPS and EEPS, indicating that AEPS had the potential to be explored as a stronger antioxidant. Furthermore, AEPS showed significant scavenging effects against $\mathrm{HO}$. at $100 \mathrm{mg} / \mathrm{L}$, with the scavenging rate reaching $81.14 \pm 3.71 \%$, which was even higher than that of EEPS $(64.37 \pm 2.53 \%)$ at $1000 \mathrm{mg} / \mathrm{L}$ (Fig. 2b). Similarly, EPS, EEPS and AEPS had obvious scavenging effects against DPPH radicals, and the scavenging activities increased with increasing concentration. The scavenging rate of AEPS reached $82.17 \pm 3.51 \%$ at $1000 \mathrm{mg} / \mathrm{L}$, which was $36.92 \pm 1.27 \%$ and $7.22 \pm 0.61 \%$ higher than those of EPS and AEPS, respectively (Fig. 2c). For the scavenging activities on $\mathrm{O}_{2}^{-}$, as exhibited in Fig. 2d, AEPS showed superior scavenging activity of $\mathrm{O}_{2}^{-}$. 
A

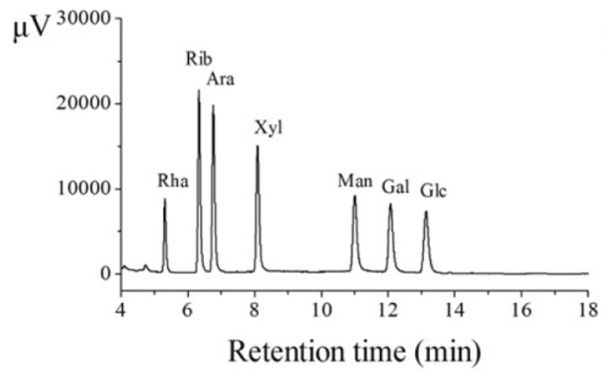

C

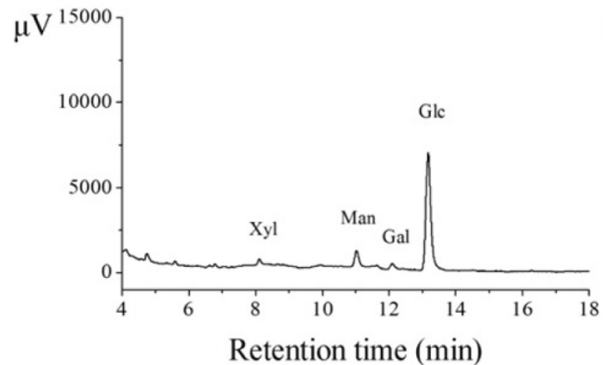

B

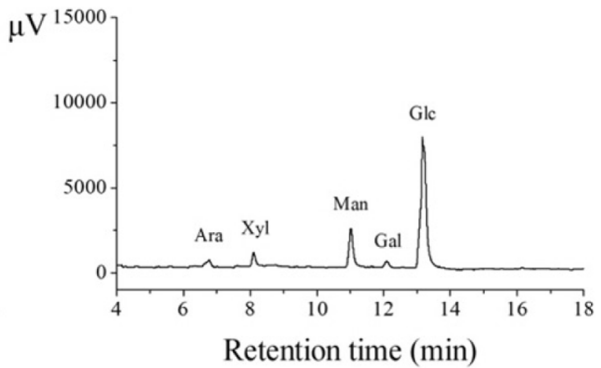

D

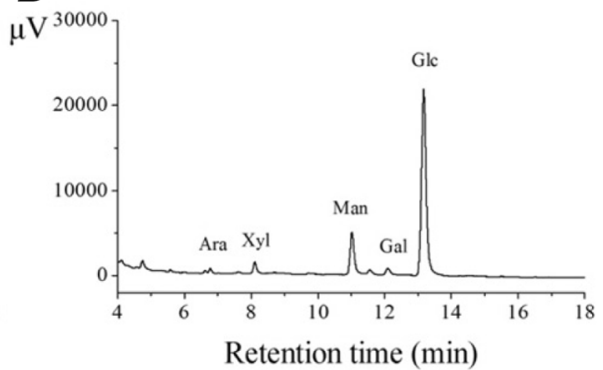

Fig. 1 Gas chromatographs of (a) standard monosaccharides, (b) EPS, (c) EEPS, and (d) AEPS

compared with EPS and EEPS in a dose-dependent manner. At $1000 \mathrm{mg} / \mathrm{L}$, the scavenging rates of AEPS, EPS and EEPS reached $80.21 \pm 2.24 \%, 65.91 \pm 3.14 \%$ and $45.23 \pm 1.75 \%$, respectively. In conclusion, the superoxide radical-scavenging activity of AEPS was stronger than those of EPS and EEPS.
Effects on body weight and the hepatosomatic index (HI)

The effects of EPS, EEPS and AEPS on body weight and the hepatosomatic index of hyperlipidaemic mice are shown in Table 1. Mice in the HL group exhibited a significant increase in body weight compared with the NC group $(P<0.001)$ on both the 10th and 20th days.




Table 1 Effects of EPS, EEPS and AEPS on body weights and $\mathrm{HI}$ of high-fat mice

\begin{tabular}{llllll}
\hline Groups & \multicolumn{2}{l}{ Body weight $(\mathrm{g})$} & \multicolumn{1}{l}{ HI (\%) } \\
\cline { 2 - 4 } & The 1st day & The 10th day & The 20th day & & The 20th day \\
\hline NC & $29.13 \pm 0.21$ & $32.19 \pm 1.69$ & $34.18 \pm 1.02$ & $3.92 \pm 0.19$ \\
HL & $29.29 \pm 0.15$ & $39.29 \pm 1.53^{\# \# \#}$ & $43.24 \pm 1.96^{\# \# \#}$ & $5.63 \pm 0.26^{\# \# \#}$ \\
ST & $29.5 \pm 0.19$ & $35.29 \pm 1.01^{* *}$ & $37.27 \pm 2.36^{* *}$ & $4.32 \pm 0.15^{* * *}$ \\
L-EPS & $28.97 \pm 0.11$ & $36.3 \pm 1.9$ & $38.96 \pm 1.55^{* *}$ & $4.95 \pm 0.12^{*}$ \\
H-EPS & $29.24 \pm 0.23$ & $36.68 \pm 1.56$ & $38.19 \pm 1.57^{* *}$ & $4.62 \pm 0.21^{* * *}$ \\
L-EEPS & $29.23 \pm 0.32$ & $36.92 \pm 1.88$ & $40.21 \pm 1.96$ & $5.43 \pm 0.16$ \\
H-EEPS & $28.96 \pm 0.17$ & $36.81 \pm 1.77$ & $38.99 \pm 1.20^{* *}$ & $5.17 \pm 0.17$ \\
L-AEPS & $29.04 \pm 0.07$ & $36.27 \pm 1.39$ & $39.02 \pm 0.92^{* *}$ & $4.78 \pm 0.23^{* *}$ \\
H-AEPS & $29.22 \pm 0.12$ & $35.01 \pm 2.21^{* *}$ & $37.21 \pm 1.30^{* * *}$ & $4.26 \pm 0.11^{* * *}$ \\
\hline
\end{tabular}

The values were reported as the mean \pm SD of eight mice per group

\#\#\#Significant difference compare to NC, $P<0.001$

*Significant difference compare to $M C, P<0.01$

**Significant difference compare to MC, $P<0.05$

***Significant difference compare to $M C, P<0.001$

Polysaccharide administrations at different doses could cause significant decreases in body weight in hyperlipidaemic mice $(P<0.05$ or $P<0.001)$ on the 20th day. Simultaneously, a significant increase in HI (liver weight/ body weight ( $\mathrm{g} / 100 \mathrm{~g}$ ) could be observed in the HL group after treatment with high-fat emulsion $(P<0.001)$. However, this uptrend could be mitigated by pre-treatment with EPS, EEPS and AEPS at 400 and $800 \mathrm{mg} / \mathrm{kg}$ body weight, indicating that they could suppress hepatic steatosis to some extent. Simvastatin had similar effects as the samples.

\section{Biochemical assays in blood serum}

The serum lipid levels, which usually contain TC, TG, HDL-C and LDL-C clinically, are shown in Table 2. After perfusion of a high-fat emulsion, the serum TG, TC and LDL-C levels of mice in the HL groups were significantly $(P<0.001$ or $P<0.05)$ increased, while the HDL-C $(P<0.05)$ levels were markedly decreased compared with the NC group, indicating successful model construction in hyperlipidaemic mice. After 20-day gavage administration, these pathologic changes were markedly mitigated by pre-treatment with EPS, EEPS and AEPS at two different dosages $(P<0.01, P<0.05$ or $P<0.001)$, respectively. As shown in Table 2, after treatment with AEPS at a dose of $800 \mathrm{mg} / \mathrm{kg}$ body weight (H-AEPS group), the TG, TC and LDL-C levels reached $1.65 \pm 0.21$, $2.29 \pm 0.06$, and $0.61 \pm 0.02 \mathrm{mM}$, lower than those of EPS $(1.80 \pm 0.15,2.89 \pm 0.13$ and $0.62 \pm 0.03 \mathrm{mM})$ and EEPS $(1.99 \pm 0.16,3.41 \pm 0.21$ and $0.81 \pm 0.05 \mathrm{mM})$, while the HDL-C levels reached $1.92 \pm 0.14 \mathrm{mM}$, higher than those of EPS $(1.82 \pm 0.05 \mathrm{mM})$ and EEPS $(1.65 \pm 0.12 \mathrm{mM})$ for the same dose groups, respectively. Treatment with simvastatin in the ST groups also showed potential effects
Table 2 Effects of EPS, EEPS and AEPS on TG, TC, HDL-C and LDL-C levels in serum

\begin{tabular}{lllll}
\hline & TG & TC & HDL-C & LDL-C \\
\hline NC & $2.14 \pm 0.11$ & $2.16 \pm 0.09$ & $1.95 \pm 0.12$ & $0.42 \pm 0.03$ \\
HL & $2.81 \pm 0.19^{\# \# \#}$ & $5.81 \pm 0.96^{\# \# \#}$ & $1.65 \pm 0.07^{\# \#}$ & $1.09 \pm 0.05^{\# \# \#}$ \\
ST & $2.09 \pm 0.21^{* * *}$ & $3.67 \pm 0.28^{* * *}$ & $2.01 \pm 0.06^{* * \#}$ & $0.56 \pm 0.04^{* * *}$ \\
L-EPS & $1.82 \pm 0.17^{* * *}$ & $3.15 \pm 0.36^{* * *}$ & $1.72 \pm 0.14$ & $0.83 \pm 0.07^{* *}$ \\
H-EPS & $1.80 \pm 0.15^{* * *}$ & $2.89 \pm 0.13^{* * *}$ & $1.82 \pm 0.05^{* *}$ & $0.62 \pm 0.03^{* * *}$ \\
L-EEPS & $2.06 \pm 0.13^{* * *}$ & $3.75 \pm 0.34^{* * *}$ & $1.68 \pm 0.13$ & $0.91 \pm 0.04^{*}$ \\
H-EEPS & $1.99 \pm 0.16^{* * *}$ & $3.41 \pm 0.21^{* * *}$ & $1.65 \pm 0.12$ & $0.81 \pm 0.05^{* *}$ \\
L-AEPS & $1.72 \pm 0.14^{* * *}$ & $2.49 \pm 0.11^{* * *}$ & $1.79 \pm 0.06^{* *}$ & $0.74 \pm 0.07^{* * *}$ \\
H-AEPS & $1.65 \pm 0.21^{* * *}$ & $2.29 \pm 0.06^{* * *}$ & $1.92 \pm 0.14^{* *}$ & $0.61 \pm 0.02^{* * *}$ \\
\hline
\end{tabular}

The values were reported as the mean \pm SD of eight mice per group

\#"Significant difference compare to NC, $P<0.05$

\#\#\# Significant difference compare to NC, $P<0.001$

*Significant difference compare to MC, $P<0.01$

**Significant difference compare to MC, $P<0.05$

***Significant difference compare to $M C, P<0.001$

against increased TG, TC and LDL-C levels and decreased HDL-C levels.

The serum enzyme activities, including ALP, ALT, AST, GSH-Px, SOD and CAT, were assayed, and the results are displayed in Fig. 3. The ALP, ALT and AST activities in the HL group were significantly higher $(P<0.001)$, while the GSH-Px, SOD and CAT activities were markedly lower than those in the NC group $(P<0.001)$, indicating that liver damage was induced by oxidative stress. Interestingly, as shown in Fig. 3, EPS, EEPS and AEPS had potential effects in decreasing high levels of ALP, ALT and AST $(P<0.01, P<0.05$ or $P<0.001)$ while increasing the low activity levels of GSH-Px, SOD and CAT at the tested dosage, respectively.

\section{Biochemical assays in liver}

The effects of EPS, EEPS and AEPS on hepatic enzyme activities, lipid peroxidation and lipid contents are shown in Fig. 4. Obvious, significant decreases in GSHPx, SOD and CAT activity levels and significant increases in MDA, LPO, TG and TC contents were observed in the HL group compared with the $\mathrm{NC}$ group, indicating that serious oxidative stress and accumulated lipids occurred in the liver $(P<0.001)$. Interestingly, these pathological changes could be alleviated by treatment with the three tested polysaccharides (EPS, AEPS and EEPS). After gavage with AEPS at the high dosage, hepatic GSH-Px and SOD activities were even higher than in the NC group. The effects on MDA and LPO contents were contrary to those for the enzyme activities. Regarding lipid contents, after 20 days of intragastric administration with AEPS, the hepatic TG levels in the L-AEPS and H-AEPS groups decreased by $27.32 \%$ and $48.29 \%$, while the TC levels reduced by $35.41 \%$ and $50.44 \%$, respectively. 

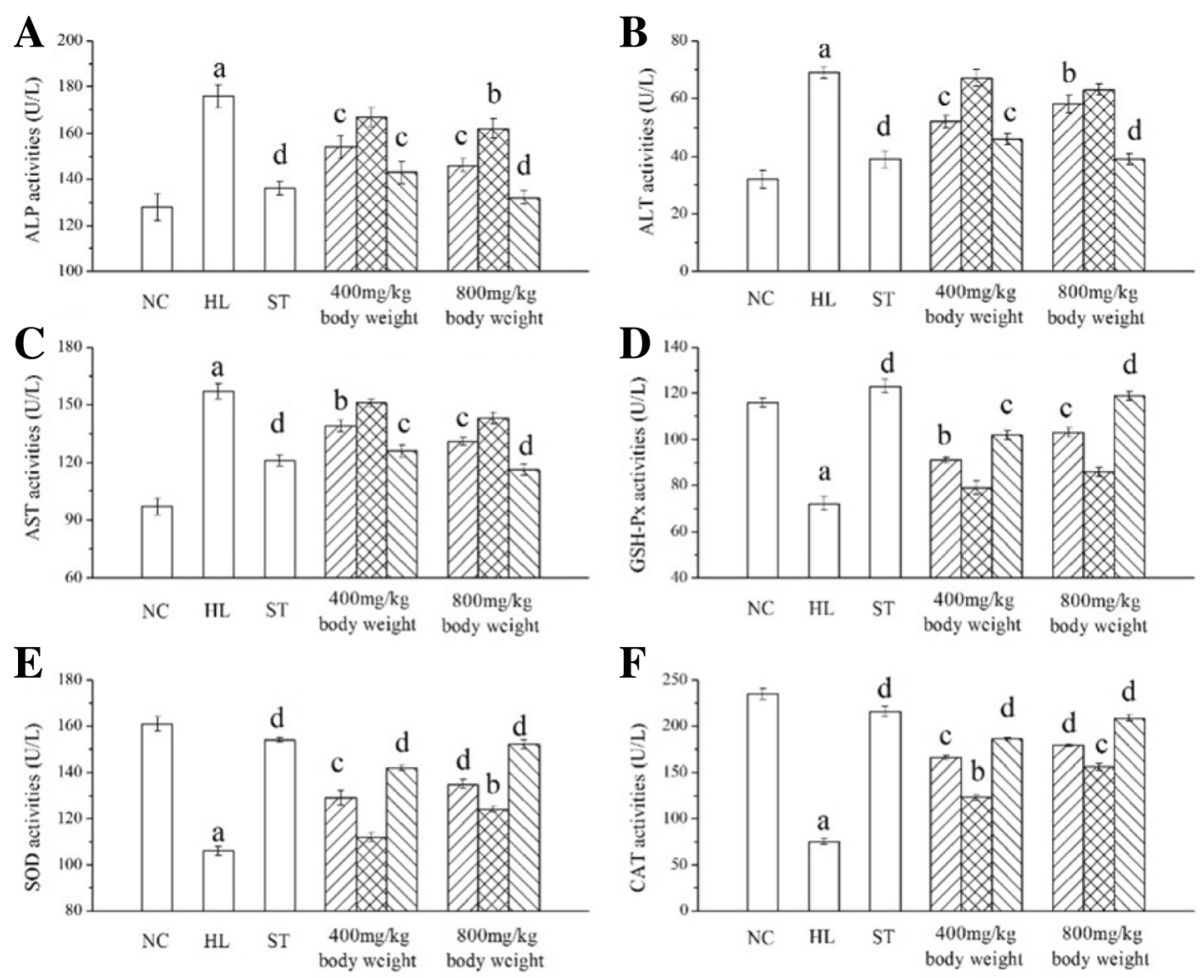

UIDEPS $\times$ EEPS MIVAEPS

Fig. 3 Effects of EPS, EEPS and AEPS on activities of (a) ALP, (b) ALT, (C) AST, (d) GSH-PX, (e) SOD and (f) CAT in serum. The values were reported as the mean \pm SD of eight mice per group. a Significant 306 difference compare to $N C, P<0.001$. $b$ Significant difference compare to MC, $P<0.01$. $C$ Significant difference compare to $M C, P<0.05$. $d$ Significant difference compare to $M C, P<0.001$

\section{Liver histopathological observation}

Hepatocyte morphological changes were observed by optical microscope and are shown in Fig. 5. The liver was badly affected by the infusion with high-fat emulsion, showing extreme swelling, diffuse hepatic steatosis, inflammatory changes, fat droplets, vesicular degeneration and disappearance of nuclei compared with normal and well-arranged cellular morphologies, including abundant cytoplasm, distinct nuclei and well-defined cell borders without any fat degeneration (Fig. 5a and b). Interestingly, morphological liver structures in the dosage groups were significantly recovered. After EPS, EEPS and AEPS treatment, fat vacuoles were markedly reduced, and hepatocyte degeneration obviously decreased. The hepatocyte morphology and arrangement in the H-AEPS group were almost normal compared with $\mathrm{NC}$ mice, indicating that AEPS had evident inhibitory effects against high-fat emulsion-induced morphologic changes and liver steatosis. Moreover, the hepatocyte morphology of mice in the ST group indicated that simvastatin had the same effects.

\section{Acute toxicity assays}

In acute toxicity assays, mice treated with EPS, EEPS and AEPS did not exhibit clinical signs of toxicity immediately or during the post-treatment period at a dosage of $5000 \mathrm{mg} / \mathrm{kg}$ body weight compared with the control group, indicating that these three polysaccharides were essentially non-toxic substances.

\section{Discussion}

The scientific literature has indicated that hyperlipidaemia plays a very important role in the developmental progress of non-alcoholic fatty liver disease, atherosclerosis and cardiovascular disease $[26,27]$. Several serum parameters, including elevated TC, TG and LDL-C levels and reduced HDL-C levels, are often considered indicators of hyperlipidaemia and are involved in increased risk of clinical diseases [28], consistent with the present results (Table 2). As the main carrier of cholesterol, excess LDL-C can be deposited in blood vessel walls, directly inducing the formation of atherosclerosis. High levels of HDL-C had protective effects because HDL-C can transport cholesterol from peripheral 

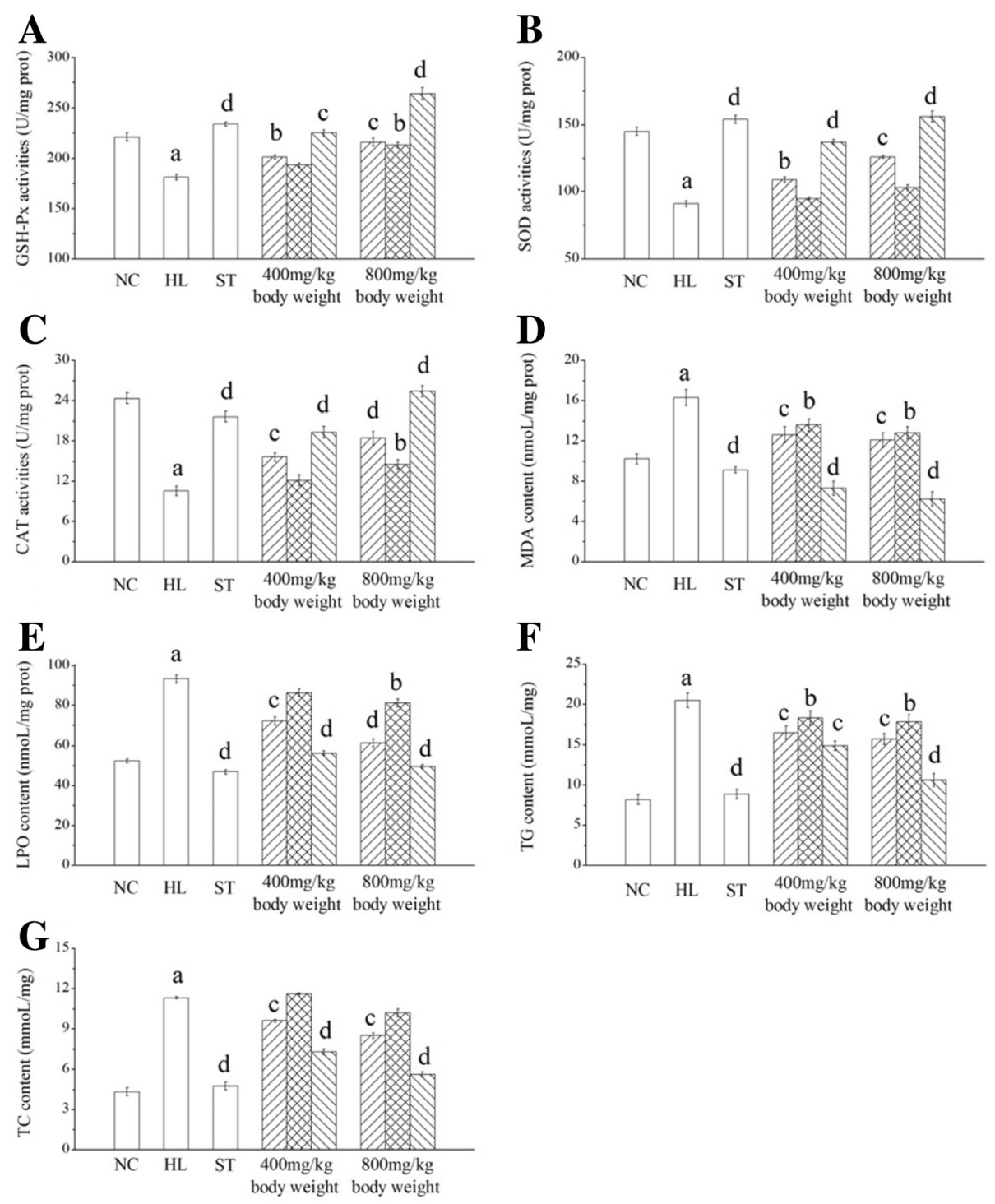

VIIA EPS

Fig. 4 Effects of EPS, EEPS and AEPS on the hepatic activities of (a) GSH-Px, (b) SOD and (c) CAT, as well as contents of (d) MDA, (e) LPO, (f) TG and (g) TC. The values were reported as the mean \pm SD of eight mice per group. The values were reported as the mean \pm SD of eight mice per group. a Significant difference compare to $N C, P<0.001$. b Significant difference compare to $M C, P<0.01$. $c$ Significant difference compare to $\mathrm{MC}, P<0.05$

tissues to the liver through the "reverse cholesterol transport" pathway for catabolism [1, 29, 30]. In addition, TG levels play key roles in the regulation of lipoprotein interactions in maintaining normal lipid metabolism and have also been proposed as major determinants of cholesterol esterification, transfer and HDL remodelling in human plasma [31]. However, the variation trends in lipid levels are significantly mitigated by treatment with these three polysaccharides (EPS, EEPS and AEPS), indicating that the polysaccharides extracted from the fermentation broth of $P$. eryngii SI-04 showed positive antihyperlipidaemic effects on restoring high-fat emulsion-induced lipid metabolic disturbance. Ren et al. [32] demonstrated that these polysaccharides might be combined with lipids in lipid metabolism, accelerating transport and excretion of serum lipids.

Previous literature has reported that oxidative stress, usually induced by ROS and motivationally accelerating the development of endothelial damage and atherosclerosis owing to its oxidative roles for the destruction of the nucleic acids, proteins and lipids of endothelial cell membranes - may be regarded as a possible mechanism to induce hyperlipidaemia [33, 34]. Lipid peroxidation could 

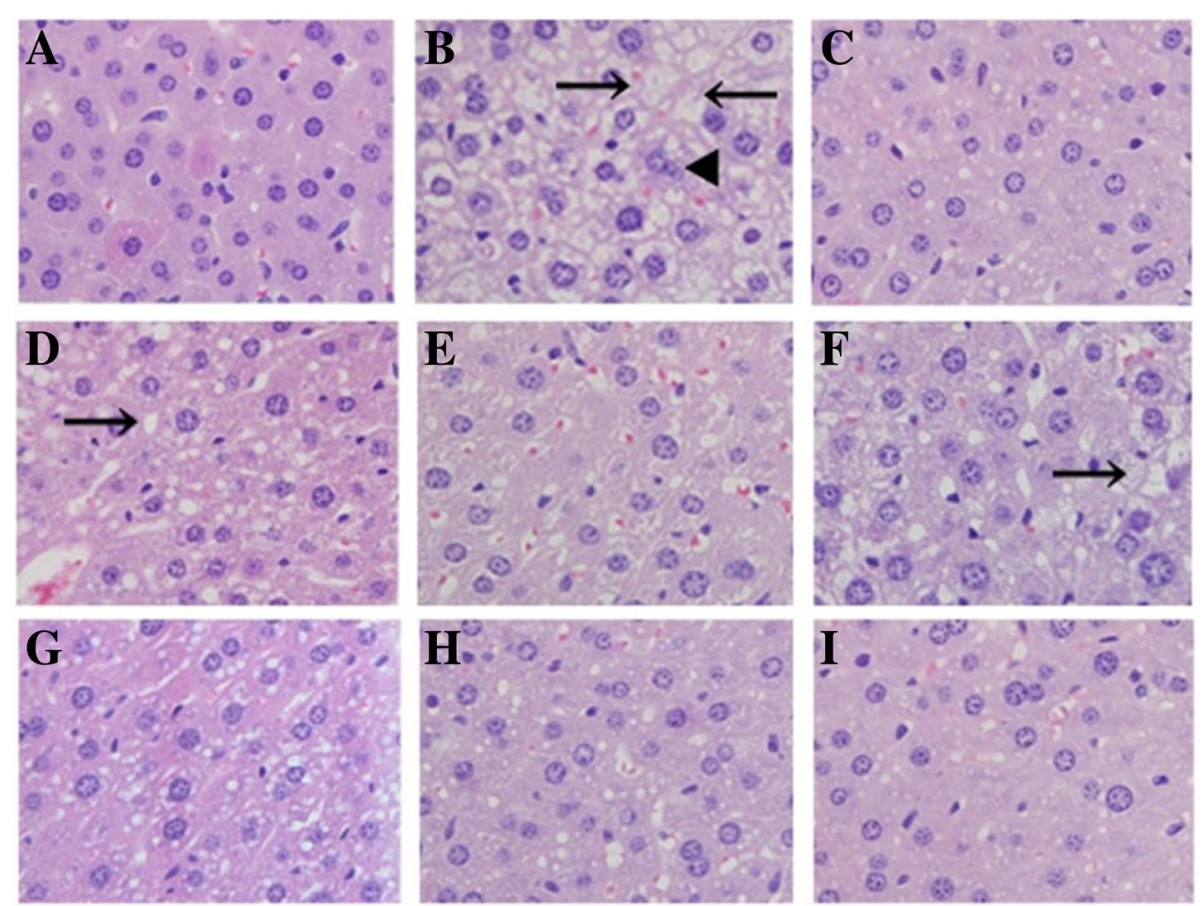

Fig. 5 Effects of EPS, EEPS and AEPS on hepatic cells in the hyperglycemia mice (hematoxylin-eosin staining, 400x). (a) NC group, (b) HL group, (c) ST group, (d) L-EPS, (e) H-EPS, (f) L-EEPS, (g) H-EEPS, (h) L-AEPS and (i) H-AEPS ( $n=8)$. Arrows show fat granule in cell, triangles indicate condensation or disappearance of cellular nucleus

be a very sensitive biomarker for investigating the antioxidant effects, since lipid peroxidation could lead to hydroperoxide generation to toxic chemicals such as MDA. Excess MDA can oxygenate and modify LDL-C to form MDA-LDL-C, which can cause the degeneration and necrosis of endothelial cells, inflammatory reactions and disordered antioxidant systems [35, 36]. Experimentally, the major antioxidant enzymes, such as SOD, GSH-Px and CAT, were commonly used as biomarkers reflecting the production of free radicals and can prevent oxidative damage cooperatively at different sites during ROS metabolic pathways [37]. In the current study, serum GSH-Px, SOD and CAT activities decreased significantly (Fig. 2) after the perfusion of high-fat emulsion. The results were in accordance with those reported in previous articles [38, 39]. The significant and dose-dependent increases in these enzyme activities after treatment with AEPS indicated that AEPS had superior activity in the treatment of hyperlipidaemia.

In addition, excessively accumulated lipids in the liver can damage hepatic biomembranes, leading to an imbalance in oxidative phosphorylation and accelerating ROS formation. The imbalance of oxidation and reduction can cause lipid peroxidation and produce significant toxic intermediate products in the liver, resulting in hepatic necrosis and apoptosis [40]. Furthermore, oxidative stress can also produce an inflammatory reaction through cell injury, causing the infiltration of the liver parenchyma by inflammatory cells [11], in accordance with the results of the hepatocyte morphological assay (Fig. 5). The hepatocytes showed obvious diffuse hepatic steatosis and inflammatory changes in the HL group, and treatment of the samples alleviated these symptoms.

Moreover, it is well known that the biological activities of polysaccharides are always associated with their monosaccharide compositions [41]. The EPS consists of five monosaccharides, including Ara, Xyl, Man, Gal and Glc, in contrast to a previous conclusion for intracellular polysaccharides (IPS) from P. eryngii SI-04 [16]. Compared with the published literature, Chen et al. [10] demonstrated that the polysaccharides of the $P$. eryngii fruit body were mainly composed of Man, Glc and Gal. The difference in monosaccharide compositions may be related to the composition of the culture medium and the fermentation, extraction and purification conditions of polysaccharides [42]. Additionally, Wu et al. [14] demonstrated that the polysaccharides showed higher biological activities after hydrolysis with various glycosidases or acidic reagents. After enzymatic and acidic hydrolysis, the monosaccharide compositions and percentage compositions of EPS were altered. The results of in vitro antioxidant and antihyperlipidaemic assays indicated that AEPS with more abundant monosaccharide compositions than EEPS and higher Glc percentages than EPS performed better in these assays (Fig. 1). 


\section{Conclusion}

In summary, EPS and its two hydrolysates (EEPS and AEPS) were successfully obtained from P. eryngii SI-04. Their antihyperlipidaemic, antioxidant and hepatoprotective activities were also investigated. AEPS exhibited potential and impressive prevention effects on high-fat dietinduced hyperlipidaemia in mice that were similar to those of the prophylactic agent simvastatin, demonstrating that polysaccharides can be exploited as potential natural drugs and functional foods for the prevention and treatment of hyperlipidaemia.

\begin{abstract}
Abbreviations
AEPS: Acidic exopolysaccharides; ALP: Alkaline phosphatase; ALT: Alamine aminotransferase; Ara: Arabinose; AST: Aspertate aminotransferase; CAT: Catalase; DPPH: 1,1-diphenyl-2-picrylhydrazyl; EEPS: Enzymatic exopolysaccharides; EPS: Exopolysaccharides; Gal: Galactose; Glc: Glucose; GSH-Px: GSH peroxide; HDL-C: high-density lipoprotein cholesterol; HI: Hepatosomatic index; IPS: Intracellular polysaccharides; LDL-C: Lowdensity lipoprotein cholesterol; LPO: Lipid peroxidation; Man: Mannose; MDA: Malondialdehyde; Rha: Rhamnose; Rib: Ribose; ROS: Reactive oxygen species; SOD: Superoxide dismutase; T-AOC: Total antioxidant capacity; TC: Total cholesterol; TG: Triacylglycerols; Xyl: Xylose
\end{abstract}

\section{Acknowledgments}

The authors gratefully acknowledge the financial supports by Mushroom Technology System of Shandong Province (SDAIT-07-05) and Fundamental Research Funds for Central Non-profit Scientific Institution (1610132016041).

\section{Funding}

The authors are grateful to the financial supports by Mushroom Technology System of Shandong Province (SDAIT-07-05) and Fundamental Research Funds for Central Non-profit Scientific Institution (1610132016041).

\section{Availability of data and materials}

The datasets used and/or analyzed during the current study will be available from the corresponding author on reasonable requests.

\section{Authors' contributions}

All experimental work and statistical analyses were performed by $C Z$ and JJZ. CZ performed most biological experiments and wrote the manuscript. JJZ performed the chemical analysis of samples. XLS, CLH and SW performed some of the biological analysis of the samples. The entire study was performed under the leadership of $L J$. JL, JW and ZYG contributed to the improvements of the English language. All authors were involved in checked the paper and contributed to the preparation of the final manuscript. All authors read and approved the final manuscript.

\section{Ethics approval and consent to participate}

All the experiments were submitted to and approved by the ethics committee of the Shandong Agricultural University.

\section{Consent for publication}

The authors declare that they consent for publication.

\section{Competing interests}

The authors declare that they have no competing interests.

\section{Publisher's Note}

Springer Nature remains neutral with regard to jurisdictional claims in published maps and institutional affiliations.

\section{Author details}

${ }^{1}$ Institute of Agricultural Resources and Environment, Shandong Academy of Agricultural Science, Key Laboratory of Wastes Matrix Utilization, Ministry of Agriculture, Jinan 250100, China. ${ }^{2}$ College of Life Science, Shandong Agricultural University, Taian 271018, China. ${ }^{3}$ Chinese Academy of
Agricultural Sciences, Beijing 100081, China. ${ }^{4}$ The Central Hospital of Taian, Taian 271000, China. ${ }^{5}$ College of Food Science, Shandong Agricultural University, Taian 271018, China. ${ }^{6}$ College of Forestry, Shandong Agricultural University, Taian 271018, China.

Received: 18 January 2017 Accepted: 26 July 2017

Published online: 14 August 2017

\section{References}

1. Zhu M, Nie P, Liang YK, Wang B. Optimizing conditions of polysaccharide extraction from Shiitake mushroom using response surface methodology and its regulating lipid metabolism. Carbohydr Polym. 2013;95(2):644-8.

2. Qiu T, Ma XJ, Ye M, Yuan RY, Wu YN. Purification, structure, lipid lowering and liver protecting effects of polysaccharide from Lachnum YM281. Carbohydr Polym. 2013;98:922-30.

3. García-Fuentes E, Gil-Villarino A, F Zafra M, García-Peregrín E. Hypocholesterolemic activity of dipyridamole: effectson chick plasma and lipoprotein composition and arachidonicacid levels. Environ Toxicol Phar. 2000;8(4):261-6.

4. Lusis AJ. Insight review article: atherosclerosis. Nature. 2000;407:233-41.

5. Ronya KA, Ajithb TA, Nimaa N, Janardhanana KK. Hypolipidemic activity of Phellinus rimosus against triton WR-1339 and high cholesterol diet induced hyperlipidemic rats. Environ Toxicol Phar. 2014;37(2):482-92.

6. Blázovics A, Fehér J, Fehér E, Kéry A, Petri G. Liver protecting and lipid lowering effects of Sempervivum tectorum extract in the rat. Phytother Res. 1993;7(1):98-100.

7. Valko M, Leibfritz D, Moncol J, Cronin MT, Mazur M, Telser J. Free radicals and antioxidants in normal physiological functions and human disease. Int J Biochem Cell B. 2007;39(1):44-84.

8. Van Herpen NA, Schrauwen-Hinderling VB. Lipid accumulation in nonadipose tissue and lipotoxicity. Physiol Behav. 2008;94(2):89-116.

9. Araujo F, Barbosa DS, Hsin CY, Maranhao RC, Abdalla DS. Evaluation of oxidative stress in patients with hyperlipidemia. Atherosclerosis. 1995; 117:61-71.

10. Chen JJ, Yong YY, Xing MC, Gu YF, Zhang Z, Zhang SZ, et al. Characterization of polysaccharides with marked inhibitory effect on lipid accumulation in Pleurotus eryngii. Carbohydr Polym. 2013;97(2):604-13

11. Zhao LY, Huang W, Yuan QX, Cheng J, Huang ZC, Ouyang LJ, et al. Hypolipidaemic effects and mechanisms of the main component of Opuntiadillenii Haw. Polysaccharides in high-fat emulsion-induced hyperlipidaemic rats. Food Chem. 2012;134(2):964-71.

12. Liu XN, Zhou B, Lin RS, Jia L, Deng P, Fan KM, Wang GY, et al. Extraction and antioxidant activities of intracellular polysaccharide from Pleurotus sp. mycelium. Int J Biol Macromol. 2010;47(2):116-9.

13. Yao ZQ, Lan J. The research progress of Pleurotus eryngii. Acta Edulis Fungi. 2004;11(1):52-8.

14. Wu T, Yan MH, Zhang Y, Miu YQ, Lu CM, Wu GY. Antioxidant and antimicrobial activity of acidolysis and enzymolysis products of fenugreek polysaccharides. J Food Sci. 2007;28(11):509-43.

15. Chen J, Mao D, Yong Y, Li J, Wei H, Lu L. Hepatoprotective and hypolipidemic effects of water-soluble polysaccharidic extract of Pleurotus eryngii. Food Chem. 2012;130(3):687-94.

16. Zhang C, Li SS, Zhang JJ, Hu CL, Che G, Zhou M, et al. Antioxidant and hepatoprotective activities of intracellular polysaccharide from Pleurotus eryngii SI-04. Int J Biol Macromol. 2016;91:568-77.

17. Yang XB, Gao XD, Han F, Xu BS, Song YC, Tan RX. Purification, characterization and enzymatic degradation of $Y C P$, a polysaccharide from marine filamentous fungus Phomaherbarum YS4108. Biochimie. 2005;87(8):747-54.

18. Chen NX, Li GY. Study on the kinetics for enzymatic degradation of a natural polysaccharide, konjac glucomannan. Macromol Symp. 2004; 216(1):165-78.

19. Ma Z, Zhang C, Gao X, Cui FY, Zhang JJ, Jia MS, et al. Enzymatic and acidic degradation effect on intracellular polysaccharide of Flammulina velutipes SF-08. Int J Biol Macromol. 2015;73:236-44.

20. Koksal E, Bursal E, Dikici E, Tozoglu F, Gulcin I. Antioxidant activity of Melissa officinalis leaves. J Med Plants Res. 2011;5(2):217-22.

21. Brand-Williams W, Cuvelier ME, Berset C. Use of a free radical method to evaluate antioxidant activity. LWT-Food Sci Technol. 1995;28(1):25-30. 
22. Kong KW, Mat-Junit S, Aminudin N, Ismail A, Abdul-Aziz A. Antioxidant activities and polyphenolics from the shoots of Barringtonia racemosa (L.) Spreng in a polar to apolar medium system. Food Chem. 2012;134(1):324-32.

23. Stewart RR, Bewley JD. Lipid peroxidation associated with accelerated aging of soybean axes. Plant Physiol. 1980;65(2):245-8.

24. Chao J, Lu TC, Liao JW, Huang TH, Lee MS, Cheng HY, et al. Analgesic and anti-inflammatory activities of ethanol root extract of Mahoniao iwakensis in mice. J Ethnopharmacol. 2009;125(2):297-303.

25. Tian L, Shi X, Yu L, Jiao Z, Rui M, Yang X. Chemical composition and hepatoprotective effects of polyphenol-rich extract from houttuynia cordata tea. J Agr Food Chem. 2012;60(18):4641-8.

26. Schwimmer JB, Pardee PE, Lavine JE, Blumkin AK, Cook S. Cardiovascular risk factors and the metabolic syndrome in pediatric nonalcoholic fatty liver disease. Circulation. 2008;118:277-83.

27. Hernandez C, Molusky M, Li Y, Li S, Lin JD. Regulation of hepatic ApoC3 expression by PGC-1 $\beta$ mediates hypolipidemic effect of nicotinic acid. Cell Metabol. 2010;12:411-9.

28. Devi R, Sharma DK. Hypolipidemic effect of different extracts of Clerodendron colebrookianum Walp in normal and high-fat diet fed rats. J Ethnopharmacol. 2004;90(1):63-8.

29. Liu X, Sun ZL, Zhang MS, Meng XM, Xia XK, Yuan WP, et al. Antioxidant and antihyperlipidemic activities of polysaccharides from sea cucumber Apostichopus japonicus. Carbohydr Polym. 2012;90(4):1664-70.

30. Wang L, Zhang Y, Xu MC, Wang YY, Cheng SJ, Liebrecht A, et al. Antidiabetic activity of Vaccinium bracteatum Thunb. Leaves' polysaccharide in STZ-induced diabetic mice. Int J Biol Macromol. 2013;61:317-21.

31. Murakami T, Michelagnoli S, Longhi R, Gianfranceschi G, Pazzucconi F, Calabresi L, et al. Triglycerides are major determinants of cholesterol esterification/transfer and HDL remodeling in human plasma. ATVB. 1995; 15(11):1819-28.

32. Ren D, Noda H, Amano H. Study on antihypertensive and antihyperlipidemic effects of marine algae. Fisheries Sci. 1994;60:423-7.

33. Jiang CX, Xiong QP, Gan D, Jiao YP, Liu J, Ma LP, et al. Antioxidant activity and potential hepatoprotective effect of polysaccharides from Cyclinasinensis. Carbohydr Polym. 2013:91:262-8.

34. Sugiura T, Dohi Y, Yamashita S, Yamamoto K, Tanaka S, Wakamatsu Y, Kimura G, et al. Malondialdehyde-modified LDL to HDL-cholesterol ratio reflects endothelial damage. Int J Cardiol. 2011;147:461-3.

35. Hao MX, Jiang LS, Fang NY, Pu J, Hu LH, Shen LH, et al. The cannabinoid WIN55, 212-2 protects against oxidized LDL-induced inflammatory response in murine macrophages. J Lipid Res. 2010;51(8):2181-90.

36. Ueda SI, Miyake I, Takata K, Shimizu N, Shirotani K, Ishida S, et al. Ezetimibe, an inhibitor of intestinal cholesterol absorption, decreases serum level of malondialdehyde-modified low-density lipoprotein in patients with hypercholesterolemia. Int J Cardiol. 2011;146(3):420-1.

37. Inal ME, Kanbak G, Sunal E. Antioxidant enzyme activities and malondialdehyde levels related to aging. Clin Chim Acta. 2001;305(1):75-80.

38. Bahramikia S, Yazdanparast R. Effect of hydroalcoholic extracts of nasturtium officinale leaves on lipid profile in high-fat diet rats. J Ethnopharmacol. 2008;115(1):116-21.

39. Chen $X$, Zhong HY, Zhu H, Zeng JH, Dai P. Effect of Curcuma kwangsiensis polysaccharides on blood lipid profiles and oxidative stress in high-fat rats. Int J Biol Macromol. 2009;44(2):138-42.

40. Kopec KL, Burns D. Nonalcoholic fatty liver disease a review of the spectrum of disease, diagnosis and therapy. Nutr Clin Pract. 2011;26(5):565-76.

41. Han Q, Yu QY, Shi J, Xiong CY, Ling ZJ, He PM. Structural characterization and antioxidant activities of 2 water-soluble polysaccharide fractions purified from tea (Camellia sinensis) flower. J Food Sci. 2011;76(3):C462-71.

42. Yin $H$, Wang $Y$, Wang $Y$, Chen $T$, Tang $H$, Wang $M$. Purification, characterization and immuno-modulating properties of polysaccharides isolated from Flammulina velutipes mycelium. Am J Chinese Med. 2010; 38(01):191-204.

\section{Submit your next manuscript to BioMed Central and we will help you at every step:}

- We accept pre-submission inquiries

- Our selector tool helps you to find the most relevant journal

- We provide round the clock customer support

- Convenient online submission

- Thorough peer review

- Inclusion in PubMed and all major indexing services

- Maximum visibility for your research

Submit your manuscript at www.biomedcentral.com/submit
C) Biomed Central 